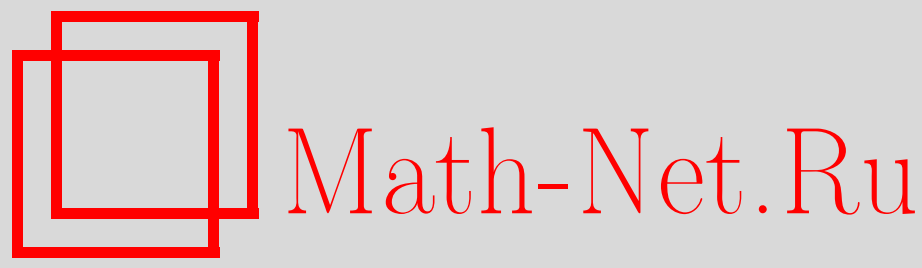

Р. Конт, Туэнь-Вай Нь, Выявление и построение эллиптического решения комплексных уравнений ГинзбургаЛандау с потенциалом в виде кубики-квинтики, ТМФ, 2012, том 172, номер 2, 224-235

DOI: https://doi.org/10.4213/tmf6953

Использование Общероссийского математического портала Math-Net.Ru подразумевает, что вы прочитали и согласны с пользовательским соглашением http://www . mathnet.ru/rus/agreement

Параметры загрузки:

IP : 35.174 .16 .151

26 апреля 2023 г., 09:23:15

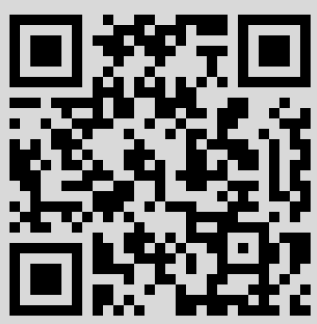


Том 172, № 2

август, 2012

(C) 2012 г. $\quad$ Р. Конт ${ }^{* \dagger}$, Туэнь-Вай Нь $^{\dagger}$

\title{
ВЫЯВЛЕНИЕ И ПОСТРОЕНИЕ ЭЛЛИПТИЧЕСКОГО РЕШЕНИЯ КОМПЛЕКСНЫХ УРАВНЕНИЙ ГИНЗБУРГА-ЛАНДАУ С ПОТЕНЦИАЛОМ В ВИДЕ КУБИКИ-КВИНТИКИ
}

\begin{abstract}
В случае уравнений движения для комплексных амлитуд оказывается, что уравнения для фазы намного сложней, чем для амплитуды. Тем не менее общие методы должны быть применимы для обеих переменных. На примере редукции комплексного уравнения Гинзбурга-Ландау для потенциала в виде кубики-квинтики с помощью движущихся волн показано, как преодолеваются трудности, возникающие при использовании двух методов: 1) критерия равенства нулю суммы вычетов эллиптического решения; 2) построения дифференциального уравнения, допускающего данное уравнение как дифференциальное следствие (метод подуравнений).
\end{abstract}

Ключевые слова: эллиптические решения, критерий вычетов, метод подуравнений, комплексное решение Гинзбурга-Ландау с потенциалом в виде кубики-квинтики.

\section{1. ВВЕДЕНИЕ. МОДУЛЬ И ФАЗА В УРАВНЕНИЯХ АМПЛИТУДЫ}

Уравнение движения $A_{t}+\cdots=0$ для комплексной амплитуды $A(x, t)$ обычно, по физическому смыслу, оказывается инвариантным относительно произвольных фазовых сдвигов $\varphi=\arg A$, где $M$ и $\varphi$ обозначают модуль и фазу, $A=M e^{i \varphi}$. Как следствие, переменная $\varphi$ входит в систему двух уравнений в частных производных для $(M, \varphi)$ только через свои производные. Тогда при редукции к обыкновенному дифференциальному уравнению (ОДУ), такой как редукция с помощью бегущих волн

$$
M \rightarrow \widetilde{M}(\xi), \quad \varphi \rightarrow-i \omega t+\widetilde{\varphi}(\xi), \quad \xi=x-c t,
$$

старший порядок дифференцирования для переменной $\psi$ в системе двух ОДУ для $\left(\widetilde{M}, \psi=\widetilde{\varphi}^{\prime}\right)$ оказывается на один меньше, чем для $M$. Следовательно, ОДУ для

${ }^{*}$ LRC MESO, Centre de mathématiques et de leurs applications et CEA-DAM, École normale supérieure de Cachan, Cachan, France. E-mail: Robert.Conte@cea.fr

${ }^{\dagger}$ Department of Mathematics, Faculty of Science, The University of Hong Kong, Hong Kong. E-mail: ntw@maths.hku.hk 
переменной $\psi$, получаемое исключением $M$, будет намного сложней (и по объему, и по структуре особенностей), чем ОДУ для $M$, получаемое исключением $\psi$.

Возьмем для примера одномерное комплексное уравнение Гинзбурга-Ландау для потенциала в виде кубики-квинтики (КГЛ5)

$$
i A_{t}+p A_{x x}+q|A|^{2} A+r|A|^{4} A-i \gamma A=0, \quad(A, p, q, r) \in \mathbb{C}, \quad p r \neq 0, \quad \operatorname{Im}(r / p) \neq 0,
$$

зависящее от семи вещественных параметров, поскольку $\gamma$ можно выбрать вещественным (сводку результатов о КГЛ5 см. в обзорах [1], [2]). Его редукция с помощью бегущих волн

$$
\begin{gathered}
A(x, t)=\sqrt{M(\xi)} e^{i(-\omega t+\varphi(\xi))}, \quad \xi=x-c t, \quad(c, \omega, M, \varphi) \in \mathbb{R}, \\
\frac{M^{\prime \prime}}{2 M}-\frac{M^{\prime 2}}{4 M^{2}}+i \varphi^{\prime \prime}-\varphi^{\prime 2}+i \varphi^{\prime} \frac{M^{\prime}}{M}-i \frac{c}{2 p} \frac{M^{\prime}}{M}+\frac{c}{p} \varphi^{\prime}+\frac{q}{p} M+\frac{r}{p} M^{2}+\frac{\omega-i \gamma}{p}=0
\end{gathered}
$$

добавляет две вещественные постоянные $(c, \omega)$, но общее количество вещественных параметров (девять) можно уменьшить до семи, сдвинув $\varphi^{\prime}$ и заметив [2], что скорость $c$ и мнимая часть $1 / p$ входят в уравнения только в виде произведения. Действительно, после обозначения восьми вещественных параметров как $e_{r}, e_{i}, d_{r}, d_{i}$, $s_{r}, s_{i}, g_{r}, g_{i}$,

$$
\begin{gathered}
e_{r}+i e_{i}=\frac{r}{p}, \quad d_{r}+i d_{i}=\frac{q}{p}, \quad s_{r}-i s_{i}=\frac{1}{p} \\
g_{r}+i g_{i}=\frac{\gamma+i \omega}{p}+\frac{c^{2} s_{r}}{4}\left(2 s_{i}+i s_{r}\right)
\end{gathered}
$$

и сдвига

$$
\varphi^{\prime}=\frac{c s_{r}}{2}+\psi
$$

система зависит только от семи вещественных параметров $e_{r}, e_{i}, d_{r}, d_{i}, g_{r}, g_{i}$, $c s_{i} \equiv \kappa_{\mathrm{i}}$.

Старшими производными в системе двух уравнений в вещественных переменных $(M, \psi)$

$$
\begin{array}{r}
\frac{M^{\prime \prime}}{2 M}-\frac{M^{\prime 2}}{4 M^{2}}-\kappa_{\mathrm{i}} \frac{M^{\prime}}{2 M}-\psi^{2}+e_{r} M^{2}+d_{r} M+g_{i}=0, \\
\psi^{\prime}+\psi \frac{M^{\prime}}{M}-\kappa_{\mathrm{i}} \psi+e_{i} M^{2}+d_{i} M-g_{r}=0
\end{array}
$$

являются $M^{\prime \prime}$ и $\psi^{\prime}$. Ниже мы воспользуемся следующей инвариантностью четности уравнения (7):

$$
\begin{aligned}
q=0: & & (M, \psi, \xi) \rightarrow(-M, \psi, \xi), \\
\kappa_{\mathrm{i}}=0: & & (M, \psi, \xi) \rightarrow(M,-\psi,-\xi) .
\end{aligned}
$$

Исключая $\psi$, получаем для $M$ одно вещественное ОДУ третьего порядка второй степени [3]

$$
\begin{gathered}
\psi=\frac{2 \kappa_{\mathrm{i}} G-G^{\prime}}{2 M^{2}\left(e_{i} M^{2}+d_{i} M-g_{r}\right)}, \quad \psi^{2}=\frac{G}{M^{2}}, \\
\left(G^{\prime}-2 \kappa_{\mathrm{i}} G\right)^{2}-4 G M^{2}\left(e_{i} M^{2}+d_{i} M-g_{r}\right)^{2}=0, \\
G=\frac{1}{2} M M^{\prime \prime}-\frac{1}{4} M^{\prime 2}-\frac{\kappa_{\mathrm{i}}}{2} M M^{\prime}+e_{r} M^{4}+d_{r} M^{3}+g_{i} M^{2},
\end{gathered}
$$


а исключая $M$, получим для $\psi$ ОДУ третьего порядка четвертой степени с 11053 членами, выпишем его доминантные члены (в смысле особенностей, см. построения в разделе 2):

$$
\begin{aligned}
e_{i}^{6} \psi^{4}\left\{\left[25 e_{i}^{3} \psi^{2} \psi^{\prime \prime \prime}-10 e_{i}^{2}\left(3 e_{i} \psi^{\prime}+4 e_{r} \psi^{2}\right) \psi \psi_{2}-6 \psi^{\prime 3} e_{i}^{3}-24 e_{r} e_{i}^{2} \psi^{\prime 2} \psi^{2}+\right.\right. \\
\left.\quad+e_{i}\left(80 e_{i}^{2}-112 e_{r}^{2}\right) \psi^{4} \psi^{\prime}-32 e_{r}\left(5 e_{i}^{2}+e_{r}^{2}\right) \psi^{6}\right]^{2}- \\
\quad-4\left[-3 e_{i}^{2} \psi^{\prime 2}+12 e_{r} e_{i} \psi^{\prime} \psi^{2}+4\left(5 e_{i}^{2}+2 e_{r}^{2}\right) \psi^{4}\right]^{2} \times \\
\left.\quad \times\left[10 e_{i}^{2} \psi \psi_{2}+e_{i}^{2} \psi^{\prime 2}+16 e_{r} e_{i} \psi^{\prime} \psi^{2}+4\left(5 e_{i}^{2}+e_{r}^{2}\right) \psi^{4}\right]\right\}^{2}+ \\
\quad+\text { субдоминантные члены }=0 .
\end{aligned}
$$

Аналогично в результате исключения переменной рациональное выражение (10) для переменной $\psi$ через $M, M^{\prime}, M^{\prime \prime}$ получается довольно простым, тогда как рациональное выражение для переменной $M$ через $\psi$ включает и третью производную $\psi^{\prime \prime \prime}$ и получается довольно длинным. Поэтому фаза комплексной амплитуды $A$ квалифицируется как "подчиненная" переменная [2], так как с ее помощью можно легко вычислить $\varphi$ из $M$, но не наоборот.

Цель настоящей работы - объяснить на приведенном выше примере, как обойти трудности, возникающие при рассмотрении $\psi$ в двух конкретных методах.

Структура статьи следующая. В разделе 2 мы изучаем детальную структуру подвижных особенностей функций $M$ и $\psi$, некоторые свойства которых ранее не отмечались. В разделе 3 мы последовательно применяем первый метод к $M$ и $\psi$, чтобы построить необходимые условия эллиптичности $M$ и $\psi$. Это оказывается легко для $M$, а для $\psi$ возникают дополнительные вопросы, которые мы далее решаем. В разделе 4 мы показываем, как правильно применять второй метод (метод подуравнений [4]-[6]) к $M$ и $\psi$, чтобы построить ОДУ первого порядка, имеющее общие эллиптические и вырожденные эллиптические решения с ОДУ (11) и (13). В разделе 5 мы просто приводим решение КГЛ 5 , в котором квадрат модуля $M$ эллиптичен. Его получение методом подуравнений будет описано в последующей работе.

\section{2. ПОДВИЖНЫЕ ОСОБЕННОСТИ КГЛ5}

В этом разделе мы перечислим все подвижные полюсы как $M$, так и $\psi$, исключая те, которые представляют собой особые решения ${ }^{1)}$ либо ОДУ третьего порядка (11) для $M$, либо ОДУ третьего порядка (13) для $\psi$.

Структура особенностей уравнения (2) изучалась в работе [8]. Первый вид особенностей $\chi=\xi-\xi_{0} \rightarrow 0$ получается балансировкой членов $A_{x x}$ и $|A|^{4} A$ в уравнении $(2)$,

$$
A \sim A_{0} \chi^{(-1 / 2+i \alpha)}, \quad \bar{A} \sim A_{0} \chi^{(-1 / 2-i \alpha)}, \quad\left(-\frac{1}{2}+i \alpha\right)\left(-\frac{3}{2}+i \alpha\right) p+A_{0}^{4} r=0
$$

1) Особое решение [7] ОДУ - это любое решение, которое нельзя получить из общего решения. Подобное решение должно сокращать нечетный множитель кратности дискриминанта ОДУ. Например, ОДУ (11) с дискриминантом $G M^{2}\left(e_{i} M^{2}+d_{i} M-g_{r}\right)^{2}$, где $G=0$, допускает в качестве особых решений все те, от которых следует отказаться, так как они не являются решениями системы (7). 
этими алгебраическими точками ветвления функции $A$ определяются четыре значения $A_{0}^{2}$ и два значения $\alpha$ :

$$
\begin{gathered}
\left(e_{i} A_{0}^{4}\right)^{2}-4 e_{r} A_{0}^{4}-3=0, \quad \alpha=\frac{e_{i}}{2} A_{0}^{4}, \\
A_{0}^{2}=\varepsilon_{2} \sqrt{\frac{2 e_{r}+\varepsilon_{1} \Delta}{e_{i}^{2}}}, \quad \alpha=\frac{2 e_{r}+\varepsilon_{1} \Delta}{2 e_{i}}, \quad \Delta=\sqrt{4 e_{r}^{2}+3 e_{i}^{2}}, \quad \varepsilon_{1,2}^{2}=1 .
\end{gathered}
$$

В этих особенностях у квадрата модуля $M=|A|^{2}$ появляются четыре простых полюса

$$
M \sim m_{0} \chi^{-1}, \quad m_{0}=A_{0}^{2},
$$

и простое исследование ОДУ третьего порядка (11) показывает, что $M$ не допускает других подвижных полюсов ${ }^{2)}$.

Посчитаем теперь полюсы $\psi$, для этого рассмотрим вещественную систему (7). Первый набор полюсов $\psi$ возникает из простых полюсов $M$,

$$
\begin{gathered}
M=m_{0} \chi_{1}^{-1}\left[1+\left(\frac{\kappa_{\mathrm{i}}}{4}+\frac{2 d_{r} m_{0}-2 e_{i} d_{i} m_{0}^{3}}{4\left(1+e_{i}^{2} m_{0}^{4}\right)}\right) \chi_{1}+O\left(\chi_{1}^{2}\right)\right], \\
\psi=\frac{e_{i} m_{0}^{2}}{2} \chi_{1}^{-1}+\frac{e_{i} m_{0}^{2}}{8} \kappa_{\mathrm{i}}+m_{0} \frac{4 d_{i}+5 e_{i} d_{r} m_{0}^{2}-e_{i}^{2} d_{i} m_{0}^{4}}{4\left(1+e_{i}^{2} m_{0}^{4}\right)}+O\left(\chi_{1}\right),
\end{gathered}
$$

в которых в силу обеих инвариантностей $(8),(9)$ требуется замена $m_{0}$ на $-m_{0}$. Этим первым набором определяются четыре разных простых полюса $\psi$ при ненулевом $q$, и только два полюса находятся иначе (в последнем случае $M^{2}$ описывается алгебраическим уравнением, допускающим два двойных полюса).

Второй набор полюсов $\psi$, не рассматривавшийся в работе [8], возникает из подвижных простых нулей $M$, и его лучше всего вычислять с помощью системы (7):

$$
\begin{aligned}
\frac{1}{M}= & \frac{1}{M_{0}} \chi_{2}^{-1}\left\{1+M_{1} \chi_{2}+\left[M_{1}^{2}+\kappa_{\mathrm{i}} M_{1}-\frac{j}{3} g_{r}+\frac{2}{3} g_{i}\right] \chi_{2}^{2}+O\left(\chi_{2}^{3}\right)\right\}, \\
\psi= & \frac{j}{2} \chi_{2}^{-1}\left\{1+\left(\kappa_{\mathrm{i}}+M_{1}\right) \chi_{2}+\left[M_{1}^{2}+2 \kappa_{\mathrm{i}} M_{1}+\frac{2}{3} g_{i}-\frac{4 j}{3} g_{r}+\frac{5}{6} \kappa_{\mathrm{i}}^{2}\right] \chi_{2}^{2}+\right. \\
& +\frac{1}{2}\left[\left(g_{r}+j g_{i}\right) \kappa_{\mathrm{i}}+\frac{3 j \kappa_{\mathrm{i}}^{3}}{4}-\frac{\left(3 d_{i}-j d_{r}\right) M_{0}}{4}+\frac{\left(11 j \kappa_{\mathrm{i}}^{2}+4 g_{r}+4 j g_{i}\right) M_{1}}{4}+\right. \\
& \left.\left.+3 j \kappa_{\mathrm{i}} M_{1}^{2}+j M_{1}^{3}\right] \chi_{2}^{3}+O\left(\chi_{2}^{4}\right)\right\},
\end{aligned}
$$

где $M_{0}$ и $M_{1}$ - произвольные постоянные, а $j$ - любой квадратный корень из -1 $(j= \pm i)$. Снова в силу обеих инвариантностей $(8)$, (9) требуется заменить $M_{0}$ на $-M_{0}$, с дополнительным условием, что $M_{1}=0$ при $\kappa_{\mathrm{i}}=0$. Этот второй набор определяет $2 N$ простых нулей $M$ и либо $2 N$ (при $q \neq 0$ ), либо $N$ (при $q=0$ ) простых полюсов $\psi$, где $N$ - неопределенное целое число.

ЗАмЕчАниЕ 1. В случае нелинейного уравнения Шредингера (где $p$ вещественно, $q$ вещественно, $r=\gamma=0$ ) из первого интеграла $M \psi=$ const следует, что все полюсы решения $\psi$ соответствуют нулям $M$.

2) Ряд Лорана $M= \pm\left(3 / 4 \cdot e_{r}\right)^{1 / 2} \chi^{-1}+\cdots$ следует исключить, так как он сокращает $G$ и, таким образом, представляет особое решение. 
Мы ищем дополнительные полюсы $\psi$, прямо исследуя ОДУ третьего порядка (13) для $\psi(\xi)$. Таким образом, мы находим три вида семейств подвижных простых полюсов: два из них только что описаны (вида (19) и (21)), а третий вид состоит из двух семейств

$$
\psi=p_{0} \chi^{-1}\left[1+\frac{\kappa_{\mathrm{i}}}{4}+O(\chi)\right], \quad 4\left(e_{r}^{2}+5 e_{i}^{2}\right) p_{0}^{2}-16 e_{r} e_{i} p_{0}+21 e_{i}^{2}=0
$$

Однако этот ряд сокращает множитель дискриминанта уравнения (13) с нечетной кратностью, поэтому это особое решение (13), и его следует исключить, поскольку оно не является решением системы (7).

Таким образом, еще одно различие между $M$ и $\psi$ состоит в разной сложности структур их особенностей: ровно четыре ряда Лорана для $M$ и неизвестное число рядов для $\psi$.

ЗАмЕчАниЕ 2. Из двух специальных значений $q=0$ и $\kappa_{\mathrm{i}}=0$, приводящих к инвариантности дифференциальной системы (см. (8), (9)), в структуре особенностей участвует только значение $q=0$. Значение $\kappa_{\mathrm{i}}=0$ появится в разделах 3 и 5 .

\section{3. О ХАРАКТЕРИЗАЦИИ НЕВЫРОЖДЕННЫХ ЭЛЛИПТИЧЕСКИХ РЕШЕНИЙ}

Согласно классическому результату внутри фундаментальной области сумма вычетов любой эллиптической функции в ее полюсах равняется нулю. Это позволило Хону [9] прибегнуть к разложениям Лорана, чтобы сгенерировать следующие необходимые условия невырожденной эллиптичности решения $u$ ОДУ:

$$
\forall j \in \mathbb{N}, \quad \forall k \in \mathbb{N}: \quad C_{j k}^{u} \equiv \sum_{\text {ряд Лорана }} \operatorname{Res}\left(\left(u^{(k)}\right)^{j}\right)=0,
$$

где сумма продолжается до любого подмножества множеств рядов Лорана, и, таким образом, выделить параметры, при которых ОДУ может иметь невырожденное эллиптическое решение.

Если предположить, что переменная $M$ эллиптична, то из (10) следует, что $\psi$ тоже эллиптична, так что можно априори воспользоваться рядом Лорана для $M$ или $\psi$, чтобы получить необходимые условия эллиптичности $M$ и $\psi$. Однако, как мы уже заметили в разделе 2 , ситуация с переменной $\psi$ гораздо сложней, чем с $M$. Действительно, некоторые разложения $\psi$ в ряд Лорана (а именно, в окрестности $\chi_{2}$ ) зависят от произвольных постоянных, поэтому необходимо сначала вычислить набор различных рядов (21), а затем решить полученные условия на вычеты для этих дополнительных произвольных постоянных. Применим критерий последовательно к переменным $M$ и $\psi$.

3.1. Критерий вычетов в применении к переменной $M$. У переменной $M$ есть два преимущества перед $\psi$ : она допускает в точности четыре разложения в ряд Лорана (18) и в эти разложения не входят никакие произвольные коэффициенты. Сумма (23) может включать один, два, три или четыре ряда Лорана. Однако в случае одного или трех рядов условие $(23)$, примененное к $\left(M^{(0)}\right)^{1}$, порождает условие $m_{0}=0$, что запрещено. В случае с двумя рядами инвариантность уравнения (15) 
относительно преобразования $m_{0} \rightarrow-m_{0}$ приводит к тому, что полученные условия совпадают с условиями для четырех рядов, поэтому достаточно изучить только один случай - когда в сумме (23) четыре ряда. Посчитав первые семь членов каждого ряда (18), получаем десять условий $C_{j k}^{M}=0, j k=01,02,03,04,05,06,07,12,13,22$,

$$
\begin{gathered}
C_{01}^{M}=0, \quad C_{02}^{M} \equiv \kappa_{\mathrm{i}} e_{r}=0, \quad C_{03}^{M} \equiv \kappa_{\mathrm{i}}\left(19 e_{r} d_{i}\left(e i^{2}+16 e_{r}^{2}\right)-e_{i} d_{r}\left(9 e i^{2}+6 e_{r}^{2}\right)\right)=0, \\
C_{04}^{M} \equiv \kappa_{\mathrm{i}} P\left(e_{r}, e_{i}, d_{r}, d_{i}, g_{r}, g_{i}, \kappa_{\mathrm{i}}^{2}\right)=0, \quad C_{12}^{M} \equiv \kappa_{\mathrm{i}} P\left(e_{r}, e_{i}, d_{r}, d_{i}, g_{r}, g_{i}, \kappa_{\mathrm{i}}^{2}\right)=0, \quad \ldots,
\end{gathered}
$$

в двух последних условиях многочлены $P$, содержат соответственно 18 и 16 членов. В случае $\kappa_{\mathrm{i}} \neq 0$ полученные ограничения имеют вид

$$
\kappa_{\mathrm{i}} \neq 0: \quad C_{02}^{M} \equiv e_{r}=0, \quad C_{03}^{M} \equiv d_{r}=0, \quad C_{04}^{M} \equiv 16 g_{i}+3 \kappa_{\mathrm{i}}^{2}=0, \quad C_{12}^{M} \equiv d_{i}=0 .
$$

В разделе 5 мы покажем, что эти необходимые условия являются достаточными, потому что существует удовлетворяющее им эллиптическое решение.

В случае $\kappa_{\mathrm{i}}=0$ в силу инвариантности четности (9) выделяется очень мало не равных тождественно нулю выражений $C_{j k}^{M}=0$, приведем первые из них ${ }^{3)}$ : $C_{13}^{M}=P_{2,2,5,5,15,15}\left(g_{r}, g_{i}, d_{r}, d_{i}, e_{r}, e_{i}\right)$ (140 членов, требуется 6 членов ряда), $C_{32}^{M}$ (не вычислено, 8 членов ряда), $C_{15}^{M}$ (не вычислено, 10 членов ряда) и т. д. Таким образом, требуется вычислить множество членов ряда, чтобы получить необходимые условия.

$\mathrm{K}$ счастью, неприятность, связанную с необходимостью иметь дело со вторым набором полюсов $\chi_{2}$ с их неизвестным количеством и добавлением произвольных коэффициентов к системе необходимых условий, можно обойти, ассоциируя с $M$ "субдоминантный” вклад “подчиненной переменной” $\psi$ следующим образом. Любое произведение $\left(\psi^{\left(k_{1}\right)}\right)^{j_{1}}\left(M^{\left(k_{2}\right)}\right)^{j_{2}}$, голоморфное вблизи $\chi_{2}$, удовлетворяет тем же свойствам, что и $M$, т. е. имеет только четыре разложения в ряд Лорана в $\chi_{1}$ и не вносит дополнительных произвольных коэффициентов.

Со случаем $\kappa_{\mathrm{i}}=0$ можно легко разобраться, простейшие необходимые условия имеют вид

$$
\begin{aligned}
\psi M: \quad & 3 e_{i}\left(2 e_{r}^{2}+3 e_{i}^{2}\right) d_{r}-e_{r}\left(8 e_{r}^{2}+11 e_{i}^{2}\right) d_{i}=0, \\
& \text { имеет решение } d_{r}=\frac{e_{r}\left(8 e_{r}^{2}+11 e_{i}^{2}\right)}{3 e_{i}\left(2 e_{r}^{2}+3 e_{i}^{2}\right)}, \\
\psi M^{2}: \quad & 32\left(2 e_{r}^{2}+3 e_{i}^{2}\right)^{2} e_{i}\left(3 e_{i} g_{i}+4 e_{r} g_{r}\right)-\left(16 e_{r}^{2}+19 e_{i}^{2}\right)\left(4 e_{r}^{2}+3 e_{i}^{2}\right) e_{r} d_{i}^{2}=0, \\
& \quad \text { имеет решение } g_{i}=-\frac{4 e_{r}}{3 e_{i}} g_{r}+\frac{\left(16 e_{r}^{2}+19 e_{i}^{2}\right)\left(4 e_{r}^{2}+3 e_{i}^{2}\right)}{96 e_{i}^{2}\left(2 e_{r}^{2}+3 e_{i}^{2}\right)^{2}} e_{r} d_{i}^{2}, \\
\psi M^{5}: \quad & d_{i} e_{r} P_{2,4,12,14}\left(g_{r}, d_{i}, e_{r}, e_{i}\right)=0, \\
\psi^{3} M^{3}: & d_{i} P_{2,4,14,16}\left(g_{r}, d_{i}, e_{r}, e_{i}\right)=0, \\
\psi M^{6}: & e_{r} P_{3,6,18,21}\left(g_{r}, d_{i}, e_{r}, e_{i}\right)=0 .
\end{aligned}
$$

\footnotetext{
3) Индексами обозначается степень $P$ по его аргументам.
} 
Всего с семью членами ряда это определяет три набора необходимых условий

$$
\begin{gathered}
\kappa_{\mathrm{i}}=0, \quad e_{r}=\frac{3 \varepsilon}{2} e_{i}, \quad d_{r}=\frac{29 \varepsilon}{15} d_{i}, \quad g_{r}=-\frac{d_{i}^{2}}{5 e_{i}}, \quad g_{i}=-\frac{7 \varepsilon d_{i}^{2}}{e_{i}}, \quad \varepsilon^{2}=1, \\
\kappa_{\mathrm{i}}=0, \quad d_{i}=d_{r}=g_{i}=g_{r}=0, \\
\kappa_{\mathrm{i}}=0, \quad d_{i}=d_{r}=g_{i}=e_{r}=0,
\end{gathered}
$$

причем последний набор - это частный случай условий $(25)$ при $\kappa_{\mathrm{i}}=0$. Первые два набора (27), (28) заведомо можно улучшить, воспользовавшись старшими членами в каждом разложении.

3.2. Критерий вычетов в применении к $\psi$. Стимулом этого исследования явилась работа [10] по приложению критерия вычетов к $\psi$, в которой найден набор необходимых условий

$$
\kappa_{\mathrm{i}}=e_{r}=d_{r}=d_{i}=g_{i} g_{r}=0,
$$

различающий только подслучай $\kappa_{\mathrm{i}}=0$ эллиптического решения (см. ниже $\left.(47)\right)$. Этот результат был получен из ряда Лорана $\psi^{j}, j=1,2,3,4$, но лишь с использованием разложений (19) вблизи $\chi_{1}$, при этом опускался второй набор полюсов (21) вблизи $\chi_{2}$. Мы покажем здесь, что, приняв во внимание оба набора полюсов $\psi$, можно получить правильные результаты, рассмативая только переменные $\left(\psi^{(k)}\right)^{j}$.

Поскольку у эллиптической функции столько же нулей, сколько и полюсов, у $M$ должно быть ровно четыре простых нуля (20). Соответствующий набор полюсов $\psi$, с необходимостью простых, примет следующий вид:

1) $q \neq 0$ : четыре полюса $\chi_{1}$ и четыре полюса $\chi_{2}$;

2) $q=0$ : два полюса $\chi_{1}$ и два полюса $\chi_{2}$.

Обозначим тройки $\left(M_{0}, M_{1}, j\right)$ в $(21)$ каждым из четырех наборов $\left(M_{0,1 \pm}, M_{1,1 \pm}, \pm i\right)$, $\left(M_{0,2 \pm}, M_{1,2 \pm}, \pm i\right)$ (случай $q \neq 0$ ) или двух наборов $\left(M_{0, \pm}, M_{1, \pm}, \pm i\right)$ (случай $q=$ $0)$. Обозначим через $C_{j k}^{\psi P}$ сумму четырех вычетов, ассоциированных с четырьмя полюсами $\chi_{1}(19)$, а через $C_{j k}^{\psi Z}-$ сумму двух вычетов, ассоциированных с двумя из полюсов $\chi_{2}(21)$ с разными значениями $j$.

Первый набор сумм вычетов дает значения [10]

$$
\begin{aligned}
C_{01}^{\psi P} \equiv & \frac{4 e_{r}}{e_{i}}, \quad C_{02}^{\psi P} \equiv \kappa_{\mathrm{i}} \frac{8 e_{r}^{2}+3 e_{i}^{2}}{2 e_{i}^{2}}, \\
C_{03}^{\psi P} \equiv 6 & \frac{e_{r}}{e_{i}} g_{i}+4 \frac{e_{r}^{2}}{e_{i}^{2}} g_{r}+\frac{9 e_{i}^{2}+32 e_{r}^{2}}{8 e_{i}^{3}} e_{r} \kappa_{\mathrm{i}}^{2}+\frac{520 e_{i}^{2} e_{r}^{4}+256 e_{r}^{6}+303 e_{i}^{4} e_{r}^{2}+9 e_{i}^{6}}{64 e_{i}^{3}\left(e_{r}^{2}+e_{i}^{2}\right)^{2}} d_{i}^{2}- \\
& \quad-\frac{132 e_{i}^{4}+229 e_{i}^{2} e_{r}^{2}+112 e_{r}^{4}}{16 e_{i}^{2}\left(e_{r}^{2}+e_{i}^{2}\right)^{2}} e_{r} d_{i} d_{r}+3 \frac{e_{i}^{2}\left(64 e_{r}^{4}+135 e_{i}^{2} e_{r}^{2}+81 e_{i}^{4}\right)}{64 e_{i}\left(e_{r}^{2}+e_{i}^{2}\right)^{2}} d_{r}^{2}, \\
C_{12}^{\psi P} \equiv \kappa_{\mathrm{i}} & {\left[g_{i}-2 \frac{e_{r}}{e_{i}} g_{r}-\frac{3}{16} \kappa_{\mathrm{i}}^{2}-\frac{112 e_{i}^{4}+23 e_{i}^{2} e_{r}^{2}-224 e_{r}^{4}}{96 e_{i}^{2}\left(e_{r}^{2}+e_{i}^{2}\right)^{2}} d_{i}^{2}\right.} \\
& \left.+\frac{132 e_{i}^{4}+229 e_{i}^{2} e_{r}^{2}+112 e_{r}^{4}}{32\left(e_{r}^{2}+e_{i}^{2}\right)^{2}} e_{r} d_{i} d_{r}+\frac{19 e_{i}^{4}-123 e_{i}^{2} e_{r}^{2}-232 e_{r}^{4}}{32 e_{i}\left(e_{r}^{2}+e_{i}^{2}\right)^{2}} d_{r}^{2}\right],
\end{aligned}
$$


а второй набор (в обозначениях $M_{1 s}=M_{1,+}+M_{1,-}, M_{1 d}=M_{1,+}-M_{1,-}$ ) дает

$$
\begin{aligned}
C_{01}^{\psi Z} \equiv & 0, \quad C_{02}^{\psi Z} \equiv-\kappa_{\mathrm{i}}-\frac{M_{1 s}}{2}, \quad C_{03}^{\psi Z} \equiv-g_{r}-\frac{3 i M_{1 d}}{4}\left(M_{1 s}+2 \kappa_{\mathrm{i}}\right) \\
C_{12}^{\psi Z} \equiv & \frac{1}{4} M_{1 s}^{3}+\frac{3}{2} \kappa_{\mathrm{i}} M_{1 s}^{2}+\frac{41}{16} \kappa_{\mathrm{i}}^{2} M_{1 s}+\frac{9}{8} \kappa_{\mathrm{i}}^{3}+\frac{3}{4}\left(2 \kappa_{\mathrm{i}}+M_{1 s}\right) M_{1 d}^{2}- \\
& \quad-i g_{r} M_{1 d}+g_{i}\left(2 \kappa_{\mathrm{i}}+M_{1 s}\right)+\frac{d_{r}}{4}\left(M_{0,+}+M_{0,-}\right)+\frac{3 i d_{i}}{4}\left(M_{0,+}-M_{0,-}\right), \\
C_{04}^{\psi Z} \equiv & \frac{5}{16} M_{1 s}^{3}+\frac{15}{8} \kappa_{\mathrm{i}} M_{1 s}^{2}+\frac{57}{16} \kappa_{\mathrm{i}}^{2} M_{1 s}+\frac{17}{8} \kappa_{\mathrm{i}}^{3}+\frac{15}{16}\left(2 \kappa_{\mathrm{i}}+M_{1 s}\right) M_{1 d}^{2}- \\
& -\frac{5}{4} i g_{r} M_{1 d}+\frac{3}{4} g_{i}\left(2 \kappa_{\mathrm{i}}+M_{1 s}\right)+\frac{d_{r}}{16}\left(M_{0,+}+M_{0,-}\right)+ \\
& +\frac{3 i d_{i}}{16}\left(M_{0,+}-M_{0,-}\right),
\end{aligned}
$$

Тогда получим набор уравнений, которые надо решить (аргументы функций $C_{j k}^{\psi Z}(\ldots)$ описывают дополнительные неизвестные):

$$
\begin{aligned}
& q \neq 0, \quad N=2: \quad C_{j k}^{\psi P}+C_{j k}^{\psi Z}\left(M_{0,1 \pm}, M_{1,1 \pm}, \pm i\right)+C_{j k}^{\psi Z}\left(M_{0,2 \pm}, M_{1,2 \pm}, \pm i\right)=0 \\
& q=0, \quad N=1: \quad \frac{1}{2} C_{j k}^{\psi P}+C_{j k}^{\psi Z}\left(M_{0, \pm}, M_{1, \pm}, \pm i\right)=0
\end{aligned}
$$

Первый набор уравнений (33) содержит слишком много неизвестных, чтобы их можно было решить, рассматривая только семь членов в каждом разложении; мы оставляем вычисления заинтересованному читателю.

Для второго набора уравнений (34), поскольку $q=0$, ряды Лорана (20), (21) должны обладать инвариантностью (8),

$$
q=0: \quad\left(M, \psi, \chi, M_{0}, M_{1}, j\right) \rightarrow\left(-M, \psi, \chi,-M_{0}, M_{1}, j\right),
$$

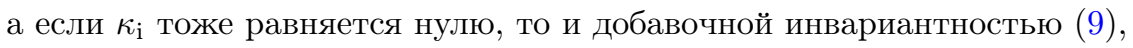

$$
q=0, \quad \kappa_{\mathrm{i}}=0: \quad M_{1}=0, \quad\left(M, \psi, \chi, M_{0}, j\right) \rightarrow\left(M,-\psi,-\chi,-M_{0}, j\right) .
$$

Второй набор уравнений дает значения

$$
\begin{aligned}
\psi^{1}: & e_{r}=0, \\
\psi^{2}: & 2 M_{1 s}+\kappa_{\mathrm{i}}=0, \\
\psi^{3}: & 8 g_{r}+9 \kappa_{\mathrm{i}} M_{1 d}=0, \\
\psi^{5}: & 128 e_{i}\left(M_{0+}^{2}+M_{0-}^{2}\right)-9 i \kappa_{\mathrm{i}} M_{1 d}\left(43 \kappa_{\mathrm{i}}^{2}+272 g_{i}\right)=0, \\
\psi^{4}: & \kappa_{\mathrm{i}}\left(3 \kappa_{\mathrm{i}}^{2}+16 g_{i}\right)=0, \\
\psi^{\prime 3} M^{3}: & M_{1 d}\left(128 i e_{i}\left(M_{0+}^{2}-M_{0-}^{2}\right)+1296 g_{i} \kappa_{\mathrm{i}}^{2}+279 \kappa_{\mathrm{i}}^{4}\right)=0,
\end{aligned}
$$

и допускает только два решения

$$
\begin{gathered}
\kappa_{\mathrm{i}} \neq 0: \quad e_{r}=0, \quad g_{i}=-\frac{3}{16} \kappa_{\mathrm{i}}^{2}, \\
M_{0 \pm}^{2}=\frac{\kappa_{\mathrm{i}}^{2}\left(16 g_{r} \pm 9 i \kappa_{\mathrm{i}}^{2}\right)}{64 e_{i}}, \quad M_{1 \pm}=\frac{-9 \kappa_{\mathrm{i}}^{2} \pm 16 i g_{r}}{36 \kappa_{\mathrm{i}}}, \\
\kappa_{\mathrm{i}}=0: \quad e_{r}=0, \quad g_{r}=0, \quad M_{1 \pm}=0, \quad M_{0+}^{2}+M_{0-}^{2}=0,
\end{gathered}
$$

поэтому подслучай $\kappa_{\mathrm{i}} \neq 0$ успешно порождает желаемые ограничения (25). 
ЗАмечАниЕ 3 . Знаменатель $\kappa_{\mathrm{i}}$ в (38) соответствует множителю $\kappa_{\mathrm{i}}$ перед членом $\psi^{4}$ в (48), и когда $\kappa_{\mathrm{i}}$ обращается в нуль, у $\chi_{2}$ больше нет особенностей в (48) (см. (49)).

Теперь, если потребовать выполнения обоих наборов необходимых условий (набоpa (25), (27)-(29) из п. 3.1 и набора (38), (39)), то остаются только две возможности существования эллиптического решения $M$ : либо (25), что определяет невырожденное эллиптическое решение, либо $e_{r}=d_{r}=d_{i}=g_{r}=g_{i}=0$, что определяет четыре рациональных решения $M=m_{0} /\left(\xi-\xi_{0}\right), \psi=\left(e_{i} m_{0}^{2} / 2\right) /\left(\xi-\xi_{0}\right)$.

\section{4. МЕТОД ОПРЕДЕЛЕНИЯ ВСЕХ ЭЛЛИПТИЧЕСКИХ И ВЫРОЖДЕННЫХ ЭЛЛИПТИЧЕСКИХ РЕШЕНИЙ}

Рассмотрим автономное алгебраическое уравнение $N$-го порядка

$$
E\left(u^{(N)}, \ldots, u^{\prime}, u\right)=0, \quad u^{\prime}=\frac{d u}{d x}
$$

допускающее по крайней мере одно разложение Лорана

$$
u=\chi^{p} \sum_{j=0}^{+\infty} u_{j} \chi^{j}, \quad \chi=x-x_{0} .
$$

Существует алгоритм [4] поиска всех его эллиптических и вырожденных эллиптических решений в явном виде. Для этого необходимо выполнить следующие последовательные шаги [5], [6].

1. Определить аналитическую структуру подвижных особенностей (например, четыре семейства простых полюсов, два двойных полюса). Для каждого подмножества семейств (например, два семейства простых полюсов) вывести эллиптические порядки $m, n$ (например, $m=2, n=4$ ) полюсов $u, u^{\prime}$ и перейти к следующим шагам.

2. Вычислить чуть больше чем $(m+1)^{2}$ членов в разложении Лорана.

3. Определить подуравнение первого порядка $m$-й степени $F\left(u, u^{\prime}\right)=0$ (оно содержит самое большее $(m+1)^{2}$ коэффициентов $\left.a_{j, k}\right)$,

$$
F\left(u, u^{\prime}\right) \equiv \sum_{k=0}^{m} \sum_{j=0}^{2 m-2 k} a_{j, k} u^{j} u^{\prime k}=0, \quad a_{0, m} \neq 0
$$

Согласно классическим результатам Брио-Буке и Пенлеве (подробнее см. [6]) любое эллиптическое и вырожденное эллиптическое решение уравнения (40) должно удовлетворять такому ОДУ, называемому “подуравнением" уравнения (40), потому что на следующем шаге потребуется принять (40) как дифференциальное следствие.

4. Потребовать, чтобы каждый ряд Лорана (41) удовлетворял уравнению $F\left(u, u^{\prime}\right)=0$,

$$
F \equiv \chi^{m(p-1)}\left(\sum_{j=0}^{J} F_{j} \chi^{j}+O\left(\chi^{J+1}\right)\right) \quad \forall j: F_{j}=0,
$$

и решить эту линейную переопределенную систему для $a_{j, k}$. 
5. Проинтегрировать каждое получившееся ОДУ $F\left(u, u^{\prime}\right)=0$.

Структура особенностей $M$ и $\psi$ была установлена в разделе 2: все подвижные полюсы $M$ и $\psi$ простые, а число различных рядов Лорана в этих простых полюсах составляет: 4 в случае $M, 4+2 N$ в случае $\psi$ или $M^{\prime} / M$, если $q \neq 0$, и $2+N$ в случае $\psi$ или $M^{\prime} / M$, если $q=0$, где $N$ - неопределенное целое число.

Начнем с переменной $M$. Для того чтобы найти все эллиптические и вырожденные эллиптические решения $M$ методом подуравнений, на шаге 1 будем рассматривать различные подмножества семейств, состоящие из одного, двух, трех или четырех рядов (18), определяющих подуравнения $F=0$ степени по $\left(M^{\prime}, M\right)$ соответственно $(1,2),(2,4),(3,6),(4,8)$. Вычисления не представляют никаких трудностей, кроме технических, их детальные результаты будут представлены в последующей публикации. Главный новый результат - невырожденное эллиптическое решение представлено в разделе 5 .

Применение метода подуравнений к переменной $\psi$ представляет определенные проблемы.

1. Главная трудность - учесть подвижные особенности типа $\chi_{2}$ (подвижные простые нули (20) для $M$, подвижные простые полюсы (21) для $\psi$ ). Именно из-за этой трудности в предшествующих исследованиях [10] удалось найти лишь особый случай эллиптического решения, представленный в разделе 5 .

2. Число разложений Лорана (21) для $\psi$ не определено, поэтому не удается установить верхнюю оценку степени подуравнения $F=0$. С этой проблемой мы уже столкнулись в п. 3.2, где было показано, что число различных рядов Лорана $\psi$ равно либо восьми $\left(q \neq 0\right.$, четыре разложения вблизи $\chi_{1}$ и четыре разложения вблизи $\left.\chi_{2}\right)$, либо четырем ( $q=0$, два разложения вблизи $\chi_{1}$ и два вблизи $\left.\chi_{2}\right)$.

3. Произвольные постоянные в разложении (21) следует определить методом подуравнений, поэтому требуется вычисление значительно большего числа членов в каждом ряде Лорана.

4. Другая трудность, априори не выявляемая, состоит в бесконечном значении $M_{1 \pm}$ при $\kappa_{\mathrm{i}}=0$ в (38). Выбор (по каким-то причинам) значения $\kappa_{\mathrm{i}}=0$ вместе с поиском подуравнений, которым удовлетворяют все четыре ряда, не приводит к успеху. В таком случае $\left(q=0\right.$ и $\left.\kappa_{\mathrm{i}}=0\right) \psi$ допускает два разложения вблизи $\chi_{1}$ и не имеет разложений вблизи $\chi_{2}$, а правильное предположение для вида подуравнения

$$
F \equiv \psi^{\prime 2}+\left(a_{01}+a_{11} \psi+a_{21} \psi^{2}\right) \psi^{\prime}+a_{00}+a_{10} \psi+a_{20} \psi^{2}+a_{30} \psi^{3}+a_{40} \psi^{4}=0
$$

что дальше редуцируется в силу классической теоремы (в $\psi^{\prime}$ нет членов первой степени (см. § 181 в [11]), поскольку $\psi$ предполагается эллиптическим) и инвариантности (9) к каноническому типу Брио-Буке

$$
F \equiv \psi^{\prime 2}+a_{00}+a_{20} \psi^{2}+a_{40} \psi^{4}=0
$$

для которого действительно решение было найдено [10] (см. ниже (49)).

По всем этим причинам, безусловно, лучше всего для уравнений амплитуды, таких как КГЛ3, КГЛ5 и других, выбирать переменную $M$. 


\section{5. ЭЛЛЛИПТИЧЕСКОЕ РЕШЕНИЕ КГЛ5}

Эллиптическим решением КГЛ5 мы называем решение КГЛ5, в котором квадрат модуля $M$ редукции с помощью бегущей волны эллиптичен.

Методом подуравнений находится единственное подуравнение рода один для $M$, и для этого требуются в точности ограничения (25). Вводя краткие обозначения

$$
e_{1}=\frac{\kappa_{\mathrm{i}}^{2}}{48}, \quad e_{0}=\frac{g_{r}}{36}
$$

перепишем это подуравнение четвертой степени как

$$
\begin{aligned}
M^{\prime 4} & -2 \kappa_{\mathrm{i}} M M^{\prime 3}+\frac{72}{e_{i}} e_{1} M^{\prime 2}\left(e_{i} M^{2}-12 e_{0}\right)+\frac{2^{4} 3^{8} e_{1}^{4}}{e_{i}^{2}}+ \\
& +\frac{648 e_{1}^{2}}{e_{i}^{2}}\left(288 e_{0}^{2}+24 e_{i} e_{0} M^{2}-e_{i}^{2} M^{4}\right)-\frac{1}{3^{4} e_{i}} M^{2}\left(e_{i} M^{2}-48 e_{0}\right)^{3}=0 .
\end{aligned}
$$

В силу упоминавшихся выше многочисленных трудностей с переменной $\psi$ соответствующее подуравнение для $\psi$ не определяется методом подуравнений, а только исключением с соответствием (10), что дает

$$
\begin{aligned}
\kappa_{\mathrm{i}} \psi^{\prime 4} & -4 \kappa_{\mathrm{i}} \psi^{\prime 3}\left(\kappa_{\mathrm{i}} \psi+24 e_{0}\right)+ \\
& +8 \psi^{\prime 2}\left(-\kappa_{\mathrm{i}}\left(27 e_{1}^{2}-324 e_{0}^{2}\right)+1440 e_{1} e_{0} \psi+27 \kappa_{\mathrm{i}} e_{1} \psi^{2}+16 e_{0} \psi^{3}+\frac{1}{3} \kappa_{\mathrm{i}} \psi^{4}\right)+ \\
& +16\left(-\frac{1}{3} \kappa_{\mathrm{i}} \psi^{8}-\frac{32}{3} e_{0} \psi^{7}-26 \kappa_{\mathrm{i}} e_{1} \psi^{6}-1632 e_{1} e_{0} \psi^{5}-\left(477 e_{1}^{2}+552 e_{0}^{2}\right) \psi^{4}-\right. \\
& -288\left(165 e_{1}^{2}+4 e_{0}^{2}\right) \psi^{3}+\kappa_{\mathrm{i}}\left(2106 e_{1}^{2}-31320 e_{0}^{2}\right) \psi^{2}+ \\
& \left.+2^{7} 3^{6}\left(e_{1}^{2}-4 e_{0}^{2}\right) e_{1} e_{0} \psi+243\left(-9 e_{1}^{4}+56 e_{1}^{2} e_{0}^{2}-144 e_{0}^{4}\right)\right)=0 .
\end{aligned}
$$

Особенность $\kappa_{\mathrm{i}}=0$, уже открывшаяся в (38), проявляется как множитель $\kappa_{\mathrm{i}}$ перед членом $\psi^{\prime 4}$ в (48), вследствие чего степень подуравнения (48) снижается с четвертой до второй при $\kappa_{\mathrm{i}}=0$. Далее можно воспроизвести результат Вернова [10]

$$
q=0, \quad e_{r}=0, \quad g_{i}=0, \quad \kappa_{\mathrm{i}}=0, \quad \begin{aligned}
& e_{i}\left(3 M^{\prime}\right)^{4}-M^{2}\left(3 e_{i} M^{2}-4 g_{r}\right)^{3}=0 \\
& 9 \psi^{\prime 2}-12 \psi^{4}-g_{r}^{2}=0
\end{aligned}
$$

где оба подуравнения для $M$ и $\psi$ принадлежат списку пяти канонических уравнений Брио-Буке.

Все детали интегрирования уравнений (47) и (48) можно найти в работе [12]. Имеем следующий окончательный результат для комплексной амплитуды $A$ :

$$
\forall \kappa_{\mathrm{i}}: \quad A=\mathrm{const} \cdot e^{-i \omega t+i c \xi / 2 p} \mathrm{H}\left(\xi,-\xi_{+}^{B}, 0\right)^{(-1+i \sqrt{3}) / 2} \mathrm{H}\left(\xi,-\xi_{-}^{B}, 0\right)^{(-1-i \sqrt{3}) / 2},
$$

где $\mathrm{H}(\xi, q, k)$ - élément simple, определенный Эрмитом ([13], т. II, с. 506) для интегрирования уравнения Ламэ,

$$
\mathrm{H}(\xi, q, k)=\frac{\sigma(\xi+q)}{\sigma(\xi) \sigma(q)} e^{(k-\zeta(q)) \xi},
$$


а фиксированные постоянные $\xi_{ \pm}^{B}$ в (50) определены уравнениями

$$
\begin{gathered}
\wp\left(\xi_{ \pm}^{B}, G_{2}, G_{3}\right)=-2 e_{1} \pm i \sqrt{3}\left(3 e_{1}+4 i e_{0}\right), \\
\wp^{\prime}\left(\xi_{ \pm}^{B}, G_{2}, G_{3}\right)=\frac{3 \mp i \sqrt{3}}{2}\left(3 e_{1}+4 i e_{0}\right), \\
G_{2}=12\left(13 e_{1}^{2}+16 e_{0}^{2}\right), \quad G_{3}=8\left(35 e_{1}^{2}+48 e_{0}^{2}\right) e_{1}, \\
\wp^{\prime 2}=4\left(\wp+2 e_{1}\right)\left(\wp^{2}-2 e_{1} \wp-35 e_{1}^{2}-48 e_{0}^{2}\right) .
\end{gathered}
$$

Численные эксперименты с периодическими граничными условиями (см. рис. 4 в работе [14]) показывают наличие решений $M$ с вещественным периодом (похожие свойства наблюдаются для КГЛ3 (рис. 7 в работе [15]), они могут соответствовать данному эллиптическому решению.

\section{6. ЗАКЛЮЧЕНИЕ}

Ловушки, описанные в настоящей статье, следует иметь в виду при поиске всех эллиптических и вырожденных эллиптических решений таких уравнений амплитуды, как комплексное уравнение Свифта-Хохенберга [16].

Благодарности. Р. Конт благодарит организаторов конференции "Solitons in $1+1$ и $2+1$ dimensions. DS, KP and all that" за приглашение и признателен за поддержку со стороны MPIPKS Dresden. Работа частично финансировалась RGC (грант № HKU 703807P).

\section{Список литературы}

[1] I. S. Aranson, L. Kramer, Rev. Mod. Phys., 74:1 (2002), 99-143, arXiv: cond-mat/0106115.

[2] W. van Saarloos, Phys. Rep., 386:2-6 (2003), 29-222, arXiv: cond-mat/0308540.

[3] A. V. Klyachkin, Modulational instability and autowaves in the active media described by the nonlinear equations of Ginzburg-Landau type, preprint № 1338, Ioffe Physical Technical Institute, Leningrad, 1989.

[4] M. Musette, R. Conte, Physica D, 181:1-2 (2003), 70-79, arXiv: nlin.PS/0302051.

[5] Р. М. Конт, М. Мюзетт, Метод Пенлеве и его приложения, НИЦ "Регулярная и хаотическая динамика", ИКИ, М., 2011.

[6] R. Conte, M. Musette, Stud. Appl. Math., 123:1 (2009), 63-81, arXiv: 0903.2009.

[7] J. Chazy, Acta Math., 34:1 (1911), 317-385.

[8] P. Marcq, H. Chaté, R. Conte, Physica D, 73:4 (1994), 305-317, arXiv: patt-sol/9310004.

[9] A. N. W. Hone, Physica D, 205:1-4 (2005), 292-306.

[10] S. Yu. Vernov, J. Phys. A, 40 (2007), 9833-9844, arXiv: nlin/0602060.

[11] C. Briot, J.-C. Bouquet, Théorie des fonctions elliptiques, 1 ed., Mallet-Bachelier, Paris, 1859; 2 ed., Gauthier-Villars, Paris, 1875.

[12] R. Conte, T. W. Ng, "Meromorphic traveling wave solutions of the complex cubic-quintic Ginzburg-Landau equation", Acta Appl. Math., 2012 (in press).

[13] G.-H. Halphen, Traité des fonctions elliptiques et de leurs applications, Gauthier-Villars, Paris, 1890, http://gallica.bnf.fr/document?O=N007348.

[14] S. Popp, O. Stiller, I. Aranson, L. Kramer, Physica D, 84:3-4 (1995), 398-423.

[15] H. Chaté, Nonlinearity, 7:1 (1994), 185-204.

[16] J. Swift, P.Cं. Hohenberg, Phys. Rev. A, 15:1 (1977), 319-328. 